\title{
Evolutionary Multi-objective Optimization of Robustness and Innovation in Redundant Genetic Representations
}

\author{
Yaochu Jin, Robin Gruna, Ingo Paenke, and Bernhard Sendhoff
}

\begin{abstract}
Robustness and innovation are two essential facets for biological evolution, where robustness means the relative insensitivity of an organism's phenotype to mutations, while innovation (evolvability) denotes the individual's ability to evolve novel phenotypes that help its survival and reproduction. Although much research has been conducted on robustness and evolvability of both biological and computational evolutionary systems, little work on the quantitative analysis of the relationship between robustness and evolvability has been reported.

In this work, a measure for innovation called local variability has been suggested. Based on a neutrality degree borrowed from literature [1] and local variability, a multi-objective evolutionary algorithm has been employed to maximize the robustness and innovation by optimizing the genotype-phenotype mapping of the redundant representation. The obtained Pareto-optimal solutions are then analyzed to reveal the trade-off relationship between robustness and innovation of the redundant representation.
\end{abstract}

\section{INTRODUCTION}

It is a challenging and extremely important task to understand how natural evolution has managed to bring about the huge biological diversity and complexity from simple particles and molecules. In addition to environmental changes, it is believed that two important principles, i.e., robustness and evolvability, may have played a central role in shaping biological diversity and complexity [2].

Biological robustness means organisms' ability to maintain relatively their functionality under a certain degree of internal and external perturbations. An important issue directly related to robustness is evolvability, which is organisms' ability to evolve inheritable novel phenotypic functionalities that help the organism survive and reproduce. In recent years, research on robustness and evolvability has become one of the main research topics in systems biology [2], [3].

Research on robustness and evolvability is still in its infancy [4], [5]. Not only a sophisticated quantitative definition for biological robustness and evolvability is still missing, but the biological origin, that is, how evolution has shaped the various biological mechanisms for robustness and evolvability remains to be understood.

In a broader sense, robustness contributes to evolvability in that without robustness, evolutionary tinkering will

Yaochu Jin and Bernhard Sendhoff are with the Honda Research Institute Europe, Carl-Legien-Str. 30, 63073 Offenbach, Germany (Email: \{yaochu.jin, bernhard.sendhoff $\}$ honda-ri.de).

Robin Gruna is with the Institut für Technische Informatik (ITEC), Universität Karlsruhe (TH), 76131 Karlsruhe, Germany (Email: gruna@ies.unikarlsruhe.de).

Ingo Paenke was with the Institut AIFB, Universität Karlsruhe (TH), 76131 Karlsruhe, Germany, and is presently with Deutsche Bahn Netz, Technology Division, 60486 Frankfurt a.M., Germany (Email: ingo.paenke@bahn.de). most likely lead to the lethal consequences, thus preventing evolution from creating new functionalities. For a clearer understanding of the mechanisms underlying evolvability and robustness, we investigate in this work evolvability in a narrow sense, that is, systems' ability to generate new phenotypes, termed innovation hereafter. Although it has been recognized qualitatively that there is a trade-off between robustness and innovation, little qualitative results have been reported with few exceptions [5].

Biological robustness can be achieved with a variety of mechanisms, such as feedback, genotypic redundancy, functional modularity, among others [3]. In the field of evolutionary computation, various methods have also been developed for the search of robust solutions [6], [7], [8], [9]. In this paper, we focus on the relationship between robustness and innovation of a redundant representation. Section II provides a mathematical definition multi-objective optimization problems. A Boolean model for redundant genotypephenotype mapping is introduced in Section III, followed by a description of three coding schemes for optimizing the model, a definition of quantitative measures for robustness and innovation ability, as well as a brief account of the multi-objective evolutionary algorithm employed to optimize the redundant representation. The experimental results are described and discussed in Section IV, where an explicit and quantitative trade-off between robustness and innovation is demonstrated and analyzed. Section V concludes the paper.

\section{Multi-OBjective Optimization}

Consider the following multi-objective minimization problem:

$$
\begin{array}{ccl}
\operatorname{minimize} & F_{m}(\mathbf{x}) & m=1,2, \cdots, M \\
\text { subject to } & g_{j}(\mathbf{x}) \geq 0, & j=1,2, \cdots, J \\
& h_{k}(\mathbf{x})=0, & k=1,2, \cdots, K \\
& x_{i}^{L} \leq x_{i} \leq x_{i}^{U}, & i=1,2, \cdots, n
\end{array}
$$

where $F_{m}(\mathbf{x})$ are the $M$ different objective functions to be minimized, $\mathbf{x}=\left(x_{1}, x_{2}, \cdots, x_{n}\right)^{T}$ is the $n$-dimensional decision space, $g_{j}(\mathbf{x})$ are the $J$ inequality constraints, $h_{k}(\mathbf{x})$ are the $K$ equality constraints, and $x_{i}^{L}$ and $x_{i}^{U}$ are the lower and upper bounds of the $i$-th decision parameter, respectively.

For the multi-objective minimization problem defined above, solution $\mathbf{x}^{(1)}$ is said to dominate solution $\mathbf{x}^{(2)}$, if $\mathbf{x}^{(1)}$ is no worse than $\mathbf{x}^{(2)}$ in all objectives, i.e.,

$$
\forall m=1,2, \cdots, M, F_{m}\left(\mathbf{x}^{(1)}\right) \leq F_{m}\left(\mathbf{x}^{(2)}\right),
$$


and if $\mathbf{x}^{(1)}$ is strictly better than $\mathbf{x}^{(2)}$ in at least one objective:

$$
\exists m^{\prime} \in\{1,2, \cdots, M\}, \text { such that } F_{m^{\prime}}\left(\mathbf{x}^{(1)}\right)<F_{m^{\prime}}\left(\mathbf{x}^{(2)}\right) .
$$

If a solution $\mathrm{x}^{*}$ is not dominated by any other feasible solutions, solution $\mathrm{x}^{*}$ is called Pareto-optimal. A set of all Pareto-optimal solutions is known as the Pareto set in the decision space, and the Pareto front in the objective space.

\section{Evolutionary Multi-ObJective Optimization of A Redundant Genotype-Phenotype Mappings}

\section{A. A Boolean Model}

Redundant representations have been widely studied in evolutionary computation [10]. In this work, we consider a genotype-phenotype mapping from $n$-dimensional genotype space $\mathcal{G} \in\{0,1\}^{n}$ to $m$-dimensional phenotype space $\mathcal{P} \in$ $\{0,1\}^{m}$, where a genotype $\mathbf{g}=\left(g_{1}, \ldots, g_{n}\right)$ is mapped to a phenotype $\mathbf{p}=\left(p_{1}, \ldots, p_{m}\right)$ as follows:

$$
p_{i}=f_{i}^{k_{i}}\left(g_{i_{1}}, \ldots, g_{i_{k_{i}}}\right)
$$

where $p_{i}, i=1,2, \ldots, m$ is the $i$-th phenotype trait, $g_{j}, j=$ $1,2, \cdots, n$ is the $j$-th gene, and

$$
\begin{aligned}
i_{1} & =\min \left\{j, j=1, \ldots, n, \forall c_{i j}=1,\right\}, \\
i_{k_{i}} & =\max \left\{j, j=1, \ldots, n, \forall c_{i j}=1,\right\},
\end{aligned}
$$

where $C=\left(c_{i j}\right)_{m \times n}$ is a binary matrix:

$c_{i j}=1: \Longleftrightarrow$ phenotype trait $p_{i}$ is affected by gene $g_{j}$, $c_{i j}=0: \Longleftrightarrow$ phenotype trait $p_{i}$ is independent of gene $g_{j}$. Thus, $f_{i}^{k_{i}}($.$) is a Boolean function with k_{i}$ inputs, where

$$
k_{i}=\sum_{j=1}^{n} c_{i j},
$$

is also known as the arity of the Boolean function.

If $k_{i}>1$, that is, if phenotype trait $p_{i}$ is influenced by more than one gene, it is called polygenic. In contrast, the number of phenotype traits affected by gene $g_{j}$ is given by the sum of the elements in the column:

$$
l_{j}=\sum_{i=1}^{m} c_{i j} .
$$

If $l_{j}>1$, then gene $g_{j}$ is said to be pleiotropic. Without loss of generality, it is assumed that $\mathbf{C}$ is chosen such that $l_{j} \geq 1$ for all $i=1, \ldots, n$. This means that each gene affects at least one phenotype trait.

The mapping can be defined by the dependencies between genes and phenotype traits, and a set of Boolean functions that determine the values of the phenotype traits. Since the connection between genotype and phenotype determines the number of inputs for a certain phenotype trait and thus for the corresponding Boolean function, the dependencies between genes and phenotype traits are determined at first. Once the connection matrix $\mathbf{C}$ has been fixed, the Boolean functions $f_{i}^{k_{i}}:\{0,1\}^{k_{i}} \rightarrow\{0,1\}, i=1 \ldots m$ can then be defined.

An example of genotype-phenotype mapping with eight genes and four phenotype traits is given in Fig. 1, where the arity of the Boolean function for the four phenotype traits is three, six, four, and two, respectively. The connection matrix in this example is as follows:

$$
\mathbf{C}=\left(\begin{array}{llllllll}
1 & 0 & 0 & 1 & 0 & 0 & 0 & 1 \\
1 & 1 & 1 & 1 & 1 & 0 & 1 & 0 \\
0 & 1 & 1 & 0 & 1 & 1 & 0 & 0 \\
0 & 0 & 1 & 0 & 0 & 0 & 1 & 0
\end{array}\right)
$$

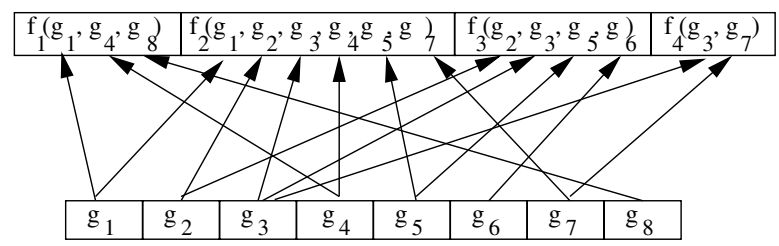

Fig. 1. An example of genotype-phenotype mapping, where the number of genes is eight, and the number of phenotype traits is four.

In the following, we are going to evolve the connection matrix $\mathbf{C}$ as well as the Boolean functions to maximize the robustness and evolvability using the NSGA-II [11], which is one of the most popular evolutionary multi-objective optimization algorithms.

\section{B. Encoding of the Boolean Model}

The first stage of the multi-objective evolutionary approach is to determine the scheme for representing the genotypephenotype mapping model. In this work, an encoding with a fixed coding length and relatively compact has been adopted.

The encoding of the Boolean model consists of two parts: the encoding of the connection matrix $C$ and the encoding of the Boolean functions $f_{i}^{k_{i}}, i=1, \ldots, m$. The encoding of $C$ is trivial, since each entry $c_{i j}$ can be written in a binary vector, leading to a fixed encoding length $m n$, independent of the actual value of $C$.

Finding an encoding for $f_{i}^{k_{i}}$ is more difficult. The connection matrix $C$ determines the polygeny $k_{i}=\sum_{j=1}^{n} c_{i j}$ of each phenotype trait $p_{i}$, i.e., the number of inputs of the Boolean function $f_{i}^{k_{i}}$. Since $f_{i}^{k_{i}}$ is a Boolean function $f_{i}^{k_{i}}:\{0,1\}^{k_{i}} \rightarrow\{0,1\}$, it is completely defined when the corresponding outputs for each $2^{k_{i}}$ inputs are determined. It can be seen that such a canonical encoding is dependent on the actual value of matrix C. During evolutionary search the size of such an encoding is changing and a set of ad hoc search operators have to be defined to guarantee that newly generated solutions are feasible.

A simpler option is to define an encoding for $f_{i}^{k_{i}}$ independent of its arity $k_{i}$. To this end, it is assumed that $k_{i}=n$ due to the fact that a phenotype trait cannot depend on more than $n$ genes. Unfortunately, this approach would lead to an encoding with a length of $n m+2^{n} m$. Even for a medium size of genotype and phenotype spaces, e.g., $m=8$ and $n=16$, the length of the chromosome will become intractably large. To address this problem, we impose more restrictions on the Boolean model so that a reasonably small encoding length can be achieved. Since the crucial part of the encoding is the encoding of the Boolean functions $f_{i}^{k_{i}}$ and its dependency 
on $k_{i}$, we are going to define a class of Boolean functions that are independent of the polygeny $k_{i}$.

Let $f^{k_{e}}$ denote an arbitrary Boolean function with arity $k_{e}$. Then the $k$-ary extension of $f^{k_{e}}, f^{k} \uparrow_{f^{k_{e}}}:\{0,1\}^{k} \rightarrow\{0,1\}$, is recursively defined by

$$
\begin{gathered}
f^{k} \uparrow_{f^{k_{e}}}\left(g_{1}, \ldots, g_{k}\right)= \\
\begin{cases}f^{k_{e}}\left(g_{1}, \ldots, g_{k_{e}}\right), & \text { if } k=k_{e} ; \\
f^{k-1} \uparrow_{f^{k_{e}}}\left(f^{k_{e}}\left(g_{1}, \ldots, g_{k_{e}}\right), g_{3}, \ldots, g_{k}\right), & \text { if } k>k_{e} ;\end{cases}
\end{gathered}
$$

if $k \geq k_{e}$, and otherwise by

$$
f^{k} \uparrow_{f^{k_{e}}}\left(g_{1}, g_{2}, \ldots, g_{k}\right)=f^{k_{e}}(\underbrace{1, \ldots, 1}_{k_{e}-k}, g_{1}, g_{2}, \ldots, g_{k}),
$$

where the first $\left(k_{e}-k\right)$ positions are set to $1 . f^{k_{e}}$ is termed the elementary Boolean function and $k_{e}$ its elementary arity. Fig. 2 shows an example of 4-ary extension of 2-ary Boolean function $f^{2}$.

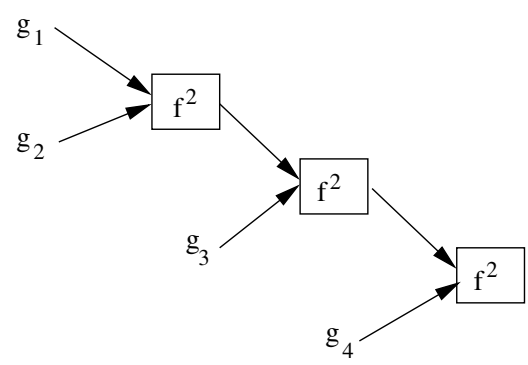

Fig. 2. An example of 4-ary extension of a 2-ary Boolean function: $f^{4} \uparrow_{f^{2}}$.

With the definition of the $k$-ary extensions $f^{k} \uparrow_{f^{k_{e}}}$ of Boolean functions, the following three different approaches to encoding the Boolean functions are considered, each of which results in a reasonably compact and fixed encoding length.

1) Multiple Boolean Functions Encoding: All $f^{k} \uparrow_{f^{k_{e}}}$ have the same encoding size $2^{k_{e}}$, independent of their actual arity $k$, since only the elementary Boolean function $f^{k_{e}}$ has to be encoded. Consequently, a restricted version of the Boolean model can be defined as follows: determine a fixed elementary arity $k_{e}$ for the model and choose every Boolean function $f_{i}=f^{k_{i}} \uparrow_{f_{i}^{k_{e}}}, i=1, \ldots m$, where $k_{i}$ is the polygeny of the corresponding phenotype trait $p_{i}$ and is determined by the connection matrix C. It is important to emphasize that each phenotype function $f_{i}$ is the $k_{i}$ ary extension of different elementary Boolean functions $f_{i}^{k_{e}}$. This model restriction leads to an encoding size of $\mathrm{nm}+$ $m 2^{k_{e}}$ bit, which is reasonably small for $k_{e}=2,3,4$. The structure of the encoding is schematically depicted below:

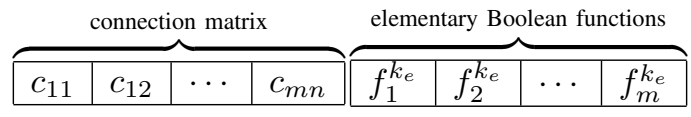

2) Single Boolean Function Encoding: An even simpler encoding can be achieved when the Boolean model is restricted to a single elementary Boolean function. In doing so, a fixed elementary arity $k_{e}$ for the model is determined and the local Boolean functions are $k_{i}$-ary extensions of the same elementary Boolean function: $f_{i}=f^{k_{i}} \uparrow_{f^{k_{e}}}, i=1, \ldots m$. This restriction leads to an encoding size of $n m+2^{k_{e}}$ bit. The structure of the encoding is schematically depicted as follows:

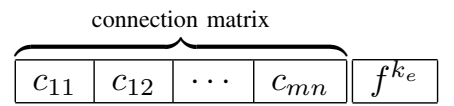

3) Majority Rule Encoding: In a further simplification of the Boolean model, we dispense with an explicit definition of the Boolean functions $f_{i}$. Instead, the phenotype traits are determined by the majority rule. There are several possibilities to break ties. In our work, we set the output of majority rule to its first input in the case of a tie. This keeps the rule balanced and deterministic:

$$
f_{i}\left(g_{j_{1}}, g_{j_{2}}, \ldots, g_{j_{k_{i}}}\right)= \begin{cases}1, & \text { if } \sum_{l=1}^{k_{i}} g_{j_{l}}>k_{i} / 2 \\ 0, & \text { if } \sum_{\substack{k_{i} \\ g_{j_{1}(i)},}} \text { otherwise. }\end{cases}
$$

Hence, only the connection matrix has to be encoded:

$$
\begin{array}{|l|l|l|l|}
\hline c_{11} & c_{12} & \cdots & c_{m n} \\
\hline
\end{array}
$$

These different encodings restrict the original model in a strong way and so one could say that each presents a different model on its own.

\section{Qualitative Measures for Robustness and Innovation}

There is no widely accepted quantitative definition for robustness and evolvability. In this work, we use local neutral degree [12], [13] for estimating the robustness and the local variability for approximating the evolvability of a genotypephenotype mapping.

Given an genotype-phenotype mapping $\phi: \mathcal{G} \rightarrow \mathcal{P}$ and a neighborhood relation $N$ over the set of genotypes $\mathcal{G}$, then the local neutral degree $\nu_{\phi}(g)$ of mapping $\phi$ is defined by [1]

$$
\nu_{\phi}(g):=\frac{\left|\left\{g^{\prime} \in \mathcal{G}: \phi(g)=\phi\left(g^{\prime}\right) \wedge N\left(g, g^{\prime}\right)\right\}\right|}{\left|\left\{g^{\prime} \in \mathcal{G}: N\left(g, g^{\prime}\right)\right\}\right|}
$$

Similar to the definition of local neutral degree, a definition of the local variability can be defined as follows. Given an genotype-phenotype mapping $\phi: \mathcal{G} \rightarrow \mathcal{P}$ and a neighborhood relation $N$ over the set of genotypes $\mathcal{G}$, then the local variability $\delta_{\phi}(g)$ of mapping $\phi$ at genotype $g$ is defined as

$$
\delta_{\phi}(g):=\frac{\left|\left\{g^{\prime} \in \mathcal{G}: \phi(g) \neq \phi\left(g^{\prime}\right) \wedge N\left(g, g^{\prime}\right)\right\}\right|}{\left|\left\{g^{\prime} \in \mathcal{G}: N\left(g, g^{\prime}\right)\right\}\right|}
$$

Note that the variability $\delta$ captures the fraction of unique phenotypes in the non-neutral neighborhood, which is accomplished by the set notation. Accordingly, $\forall g \in \mathcal{G}, \nu_{\phi}(g)+$ $\delta_{\phi}(g) \leq 1$ holds by definition. 


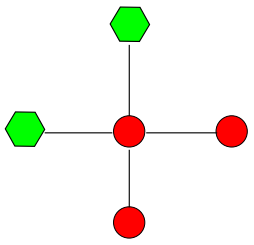

(a)

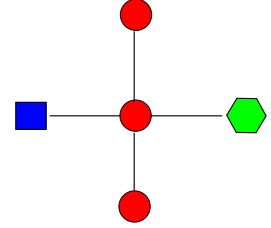

Fig. 3. Two illustrative examples on calculating local neutral degree and local variability. In the figure, filled circles, squares and pentagons denote different phenotypes, while the lines represent local neighborhood in the genotype. (a) $\nu_{\phi}(g)=0.5, \delta_{\phi}(g)=0.25$, and $(\mathrm{b}) \nu_{\phi}(g)=0.5, \delta_{\phi}(g)=$ 0.5 .

Two illustrative examples on how to calculate local neutral degree and local variability are provided in Fig. 3.

The definition of local neutral degree has been discussed in a number of studies. However, in most of these studies, the local neutral degree measure is investigated with respect to the single genotypes or neutral sets mapping to the same phenotype. In this work, we want to evaluate different characteristics of neutrality with respect to entire genotype spaces. We use the local neutral degree to calculate the mean neutral degree of a genotype-phenotype mapping, which is accomplished by randomly sampling genotypes and averaging their local neutral degree. A high mean neutral degree indicates that there are many genotypes with a high local neutral degree, and therefore the mean neutral degree reflects how many neutral mutations or how much neutrality is provided by the mapping. In the genotype space where the neutral degree is high, mutations are most likely neutral rather than deleterious. As a consequence, populations on neutral networks tend to drift toward regions with a high neutral degree to evolve robustness. This gives rise to the consideration that the mean neutral degree is related to mutational robustness.

\section{Non-Dominated Sorting and Tournament Selection}

The most significant difference of multi-objective optimization to scalar optimization is the selection method. In our work, the selection method from NSGA-II [11] is adopted, which consists of four major steps. First, the parent and offspring populations are combined. This implies that NSGAII is an elitism. Second, the combined population is sorted according to the non-dominance ranks. During the ranking, non-dominated solutions in the combined population are assigned a rank 1, which belongs to the first non-dominated front. These individuals are removed temporally from the population and the non-dominated individuals in the rest of the population are identified, which consists of the second non-dominated front of the population and are assigned a rank 2. This procedure repeats until all individuals in the combined population are assigned with a rank from 1 to $R$, assuming that $R$ non-dominated fronts can be identified in total. Third, a crowding distance reflecting the crowdedness in the neighborhood of a particular solution is calculated. The crowding distance of solution $i$ in the non-dominated front $j,(j=1, \ldots, R)$ is the distance between the two neighbors of solution $s_{i}^{j}$ in the objective space:

$$
d_{i}^{j}=\sum_{k=1}^{m}\left|F_{k}\left(s_{i-1}^{j}\right)-F_{k}\left(s_{i+1}^{j}\right)\right|,
$$

where $m$ is the number of objectives in the multi-objective optimization problem, solutions $s_{i-1}^{j}$ and $s_{i+1}^{j}$ are the two neighboring solutions of solution $s_{i}^{j}$. A large distance is assigned to the boundary solutions in each non-dominated front. Here, the larger the crowding distance, the less crowded around the solution $s_{i}^{j}$ it is. Fourth, a tournament selection which leverages between non-dominated ranking and crowdedness is conducted. Given two randomly chosen individuals, the solution with the better (lower) rank wins the tournament. If the two solutions have the same rank, the one with the larger crowding distance wins. If the two solutions have the same rank and the same crowding distance, choose a winner randomly. This procedure continues until the required number of offspring is generated.

\section{Optimization RESUlts AND Discussions}

\section{A. Experimental Results}

In the experiment, different encodings of the Boolean model were optimized with respect to the mean variability and mean neutral degree. The results for a population of 50 individuals and 100 generations are summarized in Fig. 4.

It can be seen that the different encodings lead to quite different results. The random initial population with the multiple Boolean function encoding is unevenly distributed across the objective space. The mappings lie at some distance from the true Pareto front $\bar{\nu}_{\phi}+\bar{\delta}_{\phi}=1$, albeit mappings with $\bar{\nu}>0.6$ are almost on the true Pareto front. After 100 generations, the mappings lie evenly distributed on the theoretical trade-off surface. This is different to the case in which single Boolean functions encoded. The randomly initialized population lies almost entirely on the theoretical Pareto front, but only on a section $\bar{\nu}<0.5$. After multi-objective optimization was performed, the mappings are more evenly distributed along this section. Only a few mappings lie on the Pareto front section with $\bar{\nu}<0.5$. The solutions seem to have an uniform distance from each other. For $0.2<\bar{\nu}<0.5$, however, no Pareto optimal solutions have been found. In the case of the majority rule encoding, the randomly initialized mapping form a compact cluster and evolutionary search finds Pareto optimal mappings with $0.5<\bar{\nu}<0.8$. In summary, all genotype-phenotype mappings found by different encodings of the Boolean models and evolutionary search lie on the true Pareto front $\bar{\nu}_{\phi}+\bar{\delta}_{\phi}=1$. However, the found Pareto optimal solutions are distributed along different regions. Only with the multiple Boolean function encoding was the evolutionary algorithm able to find the complete Pareto optimal front.

These results indicate that the different encodings of the Boolean functions differ in their capability of realizing various genotype-phenotype mappings. Since the multiple Boolean function encoding restricts the original Boolean model at the least, it is able to approximate the widest range 
a1

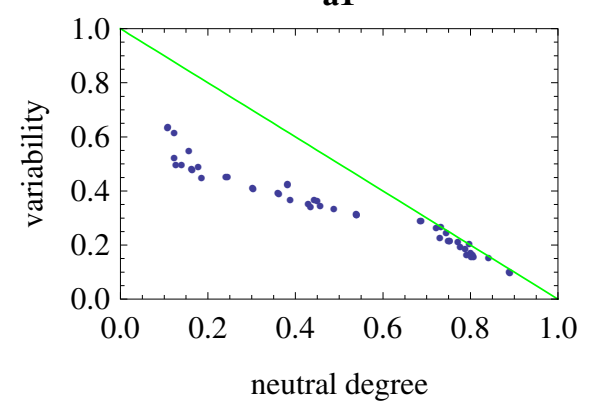

b1

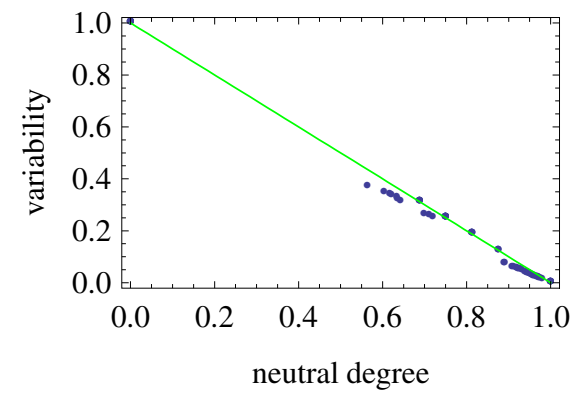

$\mathbf{c 1}$

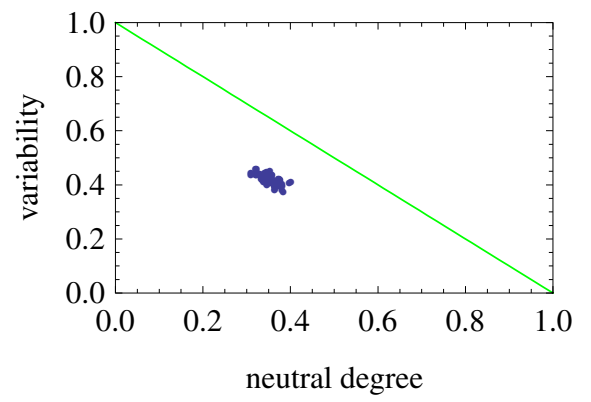

$\mathbf{a} 2$

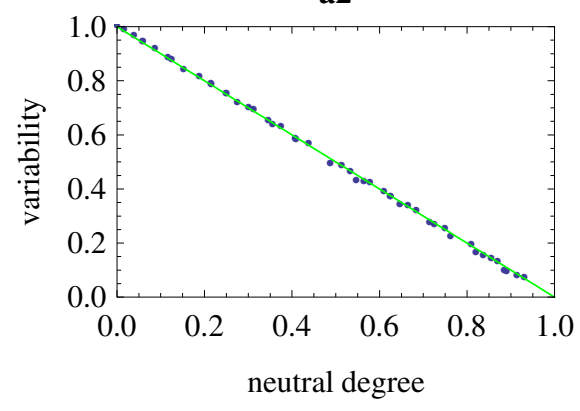

b2

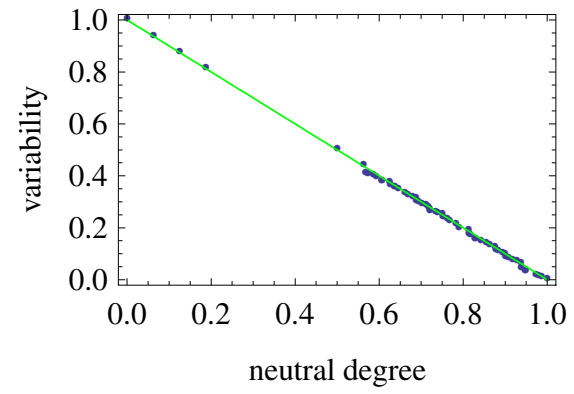

c2

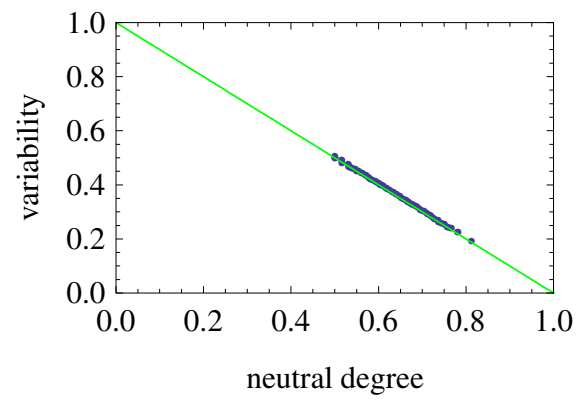

Fig. 4. Multi-objective optimization evaluation of the Boolean model $(n=16, m=8)$ for maximal mean neutral degree $\bar{\nu}_{\phi}$ and maximal mean variability $\bar{\delta}_{\phi}$. The model is encoded by different techniques: a Multiple Boolean functions encoding with elementary arity 2 . b Single Boolean function encoding with elementary arity 2. c Majority rule encoding. Plots in the left panel show the results of 50 randomly initialized genotype-phenotype mappings with $n=16$ and $m=8$. Those in the right panel show the approximated Pareto optimal set of genotype-phenotype mappings after 100 generations. The dotted line indicates the theoretical Pareto front, determined by the definition of neutral degree and variability, i. e. $\bar{\nu}_{\phi}+\bar{\delta}_{\phi}=1$. The different encodings result in different approximations of a Pareto optimal set.

of the theoretical Pareto front. For example, a genotypephenotype mapping $\phi:\{0,1\}^{16} \rightarrow\{0,1\}^{8}$ with variability $\bar{\delta}_{\phi}=1$ and neutral degree $\bar{\nu}_{\phi}=0$ can be implemented. Despite that this mapping has a redundancy of $2^{8}$, it possesses no neutrality. This means that the genotype space is structured in such a way that genotypes that map to the same phenotype are never neighbored and thus every single point mutations leads to a new phenotype. Therefore, such a mapping can be thought of as having low robustness and high evolvability.

Alternatively, mappings with neutral degree $\bar{\delta}_{\phi} \geq 0.9$ and variability $\bar{\nu}_{\phi} \leq 0.1$ can be implemented by the multiple and single Boolean functions encoding. In this case, almost all single point mutations are neutral and lead to the same phenotype. Therefore, such a mapping can be considered as highly robust and less evolvable.

\section{B. Visualization of the Connection Matrix and Boolean Function}

In an attempt to understand the Boolean model regarding the genotype-phenotype mapping as well as the Boolean functions evolved, we visualize the connection matrix and the truth value table of the Pareto-optimal models obtained when multiple Boolean functions of arity 2 are used in the model.

The connection matrix and the truth value table of the 48 Pareto-optimal solutions are illustrated in Fig. 5, where each box corresponds to one genotype-phenotype mapping model. On the left side of each box shows the $16 \times 8$ connectivity matrix, where a white grid means that the corresponding element is of value ' 0 ', and a black one denotes that the corresponding element is ' 1 '. Recall that in the connection matrix, $c_{m n}=0, m=1,2 \ldots, 8 ; n=1,2, \ldots 16$ indicates that 


\begin{tabular}{|c|c|c|c|c|c|c|c|}
\hline 78 & 81 & 28 & $9 !$ & 47 & i! & 2yz: & i! \\
\hline Pa: & 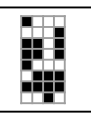 & $\because \geqslant$ & 81 & $\mathrm{BZ}:$ & 풀 & $7 \geqslant$ & 불 \\
\hline Y3: & 8 & 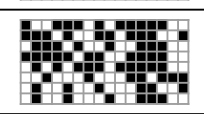 & 81 & 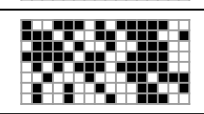 & 8 & 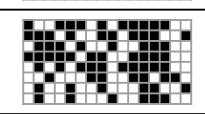 & 료 \\
\hline$\therefore 73$ & 4 & $\therefore 3$ & 4 & $\therefore 3$ & 4 & $\therefore 3$ & 4 \\
\hline 33 & 4 & 33 & 4 & 733 & 7 & 373 & 7 \\
\hline$\because 73$ & 4 & 173 & 4 & 73 & 4 & $\$ 33$ & 7 \\
\hline$\because 33$ & 7 & 783 & 4 & 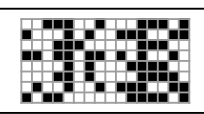 & 4 & 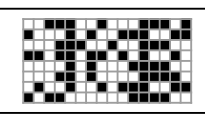 & 7 \\
\hline 73 & 4 & 763 & 4 & $3 \mathrm{Bz}$ & 4 & 76 & 4 \\
\hline$\because A 3$ & 4 & 36 & 4 & 76 & 4 & $\because A 3$ & 4 \\
\hline 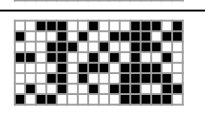 & 4 & $A 73$ & 4 & 793 & 4 & $A B$ & 7 \\
\hline $7 \mathrm{AZ}$ & 4 & Faz & 4 & 393 & 4 & 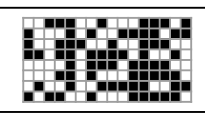 & 4 \\
\hline 783 & 7 & 193 & 4 & 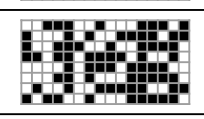 & 4 & ABZ & 7 \\
\hline 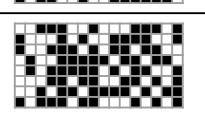 & 7 & 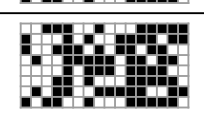 & 4 & 32 & 3 & 1328 & 7 \\
\hline 342 & 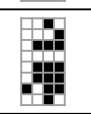 & 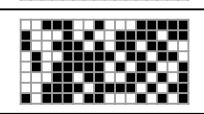 & 4 & 302 & 3 & 202 & 7 \\
\hline 338 & $\$$ & 738 & 4 & 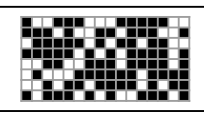 & 4 & 737 & 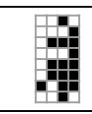 \\
\hline 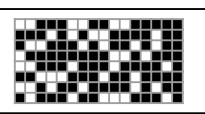 & 4 & 732 & 4 & $8+2$ & 7 & 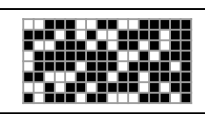 & 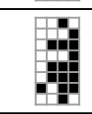 \\
\hline 732 & \# & 397 & 4 & 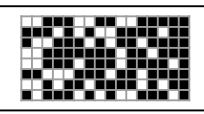 & 4 & 793 & 7 \\
\hline 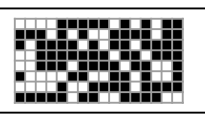 & 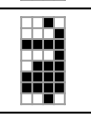 & 978 & 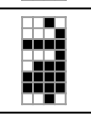 & Pat & $=$ & $7+1$ & $=$ \\
\hline
\end{tabular}

Fig. 5. Visualization of the connection matrix and the truth value table of the Boolean function of the Pareto-optimal models with multiple Boolean function of arity $2(n=16, m=8)$. The 48 solutions are ranked according to an ascending mean neutrality degree. On the left side of each box (one model) is the connection matrix, while on the right side is the truth value table of the function. In the table, a white grid denotes a ' 0 ' and a black one ' 1 '. 
phenotype trait $p_{m}$ is not directly influenced by gene $g_{n}$, and $c_{m n}=1$ indicates that phenotype trait $p_{m}$ is directly influenced by gene $g_{n}$. Therefore, the pleiotropy of gene $g_{n}$ is given by the sum of column $n$, whereas the polygeny of phenotype trait $p_{m}$ is given by the sum of row $m$. On the right side of each box shows the truth value of the Boolean function of arity 2, where the $m$-row depicts the table of truth value of the elementary Boolean function $f_{m}^{2}$.

From Fig. 5, we are not able to observe a clear regularity in the structure of the connection matrix, which may be attributed to the fact that robustness and innovation of the Boolean models with multiple Boolean functions is a combined result of connectivity and the Boolean function.

\section{Uniformity of Pareto Optimal Genotype-Phenotype Map- pings}

We further analyze the Pareto-optimal mappings obtained by the evolutionary algorithm by investigating the uniformity of the genotype-phenotype mappings. A genotype-phenotype mapping is uniformly redundant, if all accessible phenotypes are represented by the same number of genotypes. In the literature there is no direct statements about the relationship between uniformity and evolvability, nor between uniformity and robustness. It is believed, however, that uniformity is an important issue when designing redundant genotypephenotype mappings. Here, the uniformity statistic introduced by Ebner et al. [14] is adopted to estimate whether a genotype-phenotype mapping is uniformly redundant or not. At first, the probability of locating any of the phenotypes in the solution space using random search is estimated, which is accomplished by randomly sampling genotypes from genotype space and determining the corresponding phenotypes. Then the relative frequency of each phenotype was displayed as a histogram. Therefore the histogram summarizes the sizes of the neutral sets of all phenotypes. The shape of the estimated probability distribution reflects the uniformity of the genotype-phenotype mapping. If the distribution is flat, all phenotypes are represented by the same number of genotypes and the genotype-phenotype mapping is uniformly redundant. In the case of a highly uneven distribution, there are phenotypes which cover an excessive amount of the genotype space and any search would be strongly biased toward these phenotypes. Furthermore, if the genotype-phenotype mapping is not surjective, the histogram shows phenotypes with probability zero. These phenotypes do not lie in the image of the mapping and can never be reached. It should be mentioned that there is no natural ordering of the genotypes and therefore the ordering of the genotypes in the histogram is arbitrary. In this work, genotypes are represented as binary vectors of a given length, we thus use the ordering of binary numbers, starting with the all-zero genotype.

In the analysis, we examine the Pareto optimal solutions when multiple Boolean functions encoding is used, in which case a most complete Pareto optimal set of mappings has been generated. The histogram representations of uniformity of the 48 Pareto-optimal solutions are plotted in Fig. 6 (a). In addition, the entropy of the estimated phenotype distribution was calculated and compared with the neutral degree and variability of the Pareto optimal mappings, both of which are depicted in Fig. 6. The histogram representation of uniformity shows that almost all Pareto optimal mappings represent phenotypes by different numbers of genotypes. With an increasing neutral degree, more and more phenotypes have probability zero to be located by random search. In other words, those Pareto-optimal models with a high degree of neutrality map the whole genotype space to only few phenotypes. This can also be seen in more detail when the normed entropy of the phenotype distribution is considered. Up to a neutral degree $\bar{\nu}_{\phi}=0.5$ the entropy is relatively high with values between 0.8 and 1 . For a neutral degree $\bar{\nu}_{\phi}>0.5$, the entropy decreases almost linearly to 0.1 .

The results suggest that the mean neutral degree is directly related to the uniformity of a genotype-phenotype mapping. It seems that the genotype-phenotype mappings implemented by the proposed model can only provide a high entropy up to a certain neutral degree threshold. Fig. 6 illustrates that with an increasing degree of neutrality, entropy decreases and less and less phenotypes lie in the image of the mappings. This can be seen in the histogram representation of the uniformity estimation, where for a higher neutral degree and lower entropy, more and more phenotypes have a probability of zero. This observation is interesting in the light of interpreting the mean neutral degree as robustness to genetic variations. The mapping with the highest robustness would then be the constant mapping that maps the whole genotype space to a single phenotype. There is no genetic variation that could ever alter the phenotype. In this case, no phenotypic adaptation is possible and thus the mapping offers no evolvability.

\section{CONCLUSiOns}

An evolutionary multi-objective optimization algorithm has been employed to maximize the robustness (local neutrality) and innovation (local variability) for a class of redundant Boolean representations. In this way, an explicit and quantitative trade-off relationship between robustness and innovation can be revealed. Such trade-off relationships indicate that there is no single representation that can reach a maximum of robustness and innovation at the same time. An optimal representation then depends on the environmental condition: if the environment is changing rapidly, a representation with a higher innovation ability might be preferred to enhance the survival probability, whereas in case of a slowing changing environment, stronger robustness may provide the system a chance to reach a higher fitness.

Many interesting issues remain to be investigated. On the one hand, quantitative measures other than the local neutrality and local variability [15] can be considered for measuring robustness and innovation. In addition, similar optimization and analysis can be extended to more complex, in particular, dynamic genotype-phenotype mappings described e.g. by random Boolean networks or ordinary differential equations. 

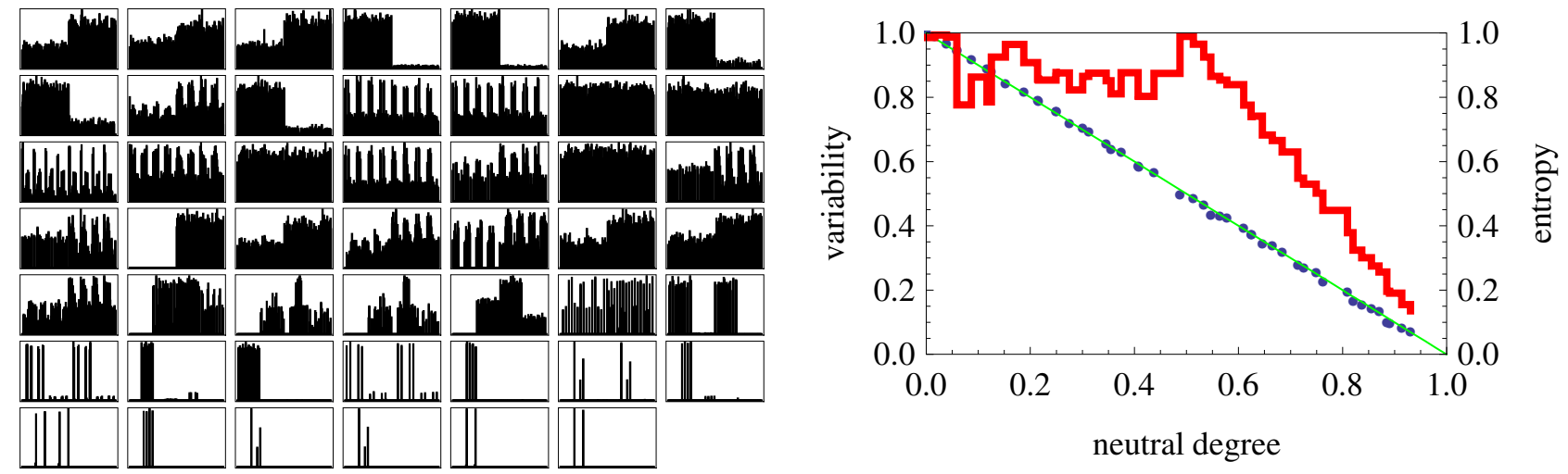

Fig. 6. Uniformity of Pareto optimal Boolean rule mappings $(n=16, m=8)$ with respect to mean neutral degree and mean variability, encoded with multiple Boolean functions and elementary arity 2. Left panel: Histogram representation of uniformity of the 48 Pareto-optimal models. Each histogram corresponds to a mapping lying on the Pareto front. This statistic indicates the amount of genotype space covered by different phenotypes. The horizontal axis represents the phenotype space, while the vertical axis indicates the relative frequency of each phenotype. The axises are omitted in the plots. The histograms are sorted by an ascending mean neutral degree of the corresponding mapping, starting with from the upper left to the lower right. With an ascending neutral degree, the number of accessible phenotypes decreases. Right panel: Entropy of the estimated phenotype distribution shown on the left. The Pareto optimal mappings are depicted together with their corresponding entropy with the thick line. The entropy decreases as the degree of neutrality increases.

\section{REFERENCES}

[1] P. K. Lehre and P. C. Haddow, "Phenotypic complexity and local variations in neutral degree," BioSystems, vol. 87, pp. 233-242, Feb. 2007. [Online]. Available: http://www.sciencedirect.com/science/article/B6T2K-4KVJCK68/2/099604f1791eecd1e23f7ea94729a5a9

[2] A. Wagner, Robustness and evolvability in living systems. Princeton University Press, 2007.

[3] H. Kitano, "Biological robustness." Nat Rev Genet, vol. 5, no. 11, pp. 826-37, 2004.

[4] R. Lenski, J. Barrick, and C. Ofria, "Balancing robustness and evolvability," PLOS Biology, vol. 4, no. 2, p. e428, 2006.

[5] S. Ciliberti, O. Martin, and A. Wagner, "Innovation and robustness in complex regulatory gene networks," Proceedings of National Academy of Science of USA, vol. 104, pp. 13 591-13 596, 2007.

[6] Y. Jin and J. Branke, "Evolutionary optimization in uncertain and dynamic environments - A survey," IEEE Transactions on Evolutionary Computation, vol. 9, no. 3, pp. 303-317, 2005.

[7] N. Nicosia, S. Rinaudo, and E. Sciacca, "An evolutionary algorithmbased approach to robust circuit design using constrained multiobjective optimization," Knowledge Based Systems Journal, vol. 21, no. 3, pp. 175-183, 2008.

[8] I. Paenke, J. Branke, and Y. Jin, "Efficient search for robust solutions by means of evolutionary algorithms and fitness approximation," IEEE Transactions on Evolutionary Computation, vol. 10, no. 4, pp. 405420, 2006.

[9] S. Tsutsui and A. Ghosh, "Genetic algorithms with a robust solution search scheme," IEEE Transactions on Evolutionary Computation, vol. 1, no. 3, pp. 201-208, 1997.

[10] F. Rothlauf, Representations for Genetic and Evolutionary Algorithms. Heidelberg: Springer, 2006.

[11] K. Deb, S. Agrawal, A. Pratap, and T. Meyarivan, "A fast elitist nondominated sorting genetic algorithm for multi-objective optimization: NSGA-II," in Parallel Problem Solving from Nature, vol. VI, 2000, pp. 849-858.

[12] M. Kimura, The Neutral Theory of Molecular Evolution. Cabridge University Press, 1983.

[13] E. van Nimwegen, J. Crutchfield, and M. Huynen, "Neutral evolution of mutational robustness," PNAS, pp. 9716-9720, 1999.

[14] M. Ebner, P. Langguth, J. Albert, M. Schakleton, and R. Shipman, "On neutral networks and evolvability," in Congress on Evolutionary Computation. IEEE Press, 2001, pp. 27-30.

[15] R. Gruna, "Analysis of redundant genotype-phenotype mappings Investigation of the effect of neutrality on evolvability and robustness," Master's thesis, AIFB, Universität Karlsruhe, 2007. 